\title{
Transmembrane-4 L-six family member-1 (TM4SF1) promotes non-small cell lung cancer proliferation, invasion and chemo- resistance through regulating the DDR1/ Akt/ERK-mTOR axis
}

Lin Ye ${ }^{1,2}$, Chunyun Pu ${ }^{2}$, Jun Tang ${ }^{2}$, Yan Wang ${ }^{2}$, Can Wang ${ }^{3}$, Zhu Qiu², Tingxiu Xiang ${ }^{2}$, Yunmei Zhang ${ }^{4^{*}}$ and Weiyan Peng ${ }^{2 *}$

\begin{abstract}
Background: Tumor chemo-resistance is a hallmark of malignant tumors as well as the major cause of poor survival rates in lung cancer. Transmembrane-4 L-six family member-1 (TM4SF1), an antigen that serves as an oncogene, mainly affects tumor invasion and metastasis. We investigated the roles of TM4SF1 in non-small-cell lung cancer progression, particularly in the regulation of chemo-sensitivity.

Methods: TMASF1 was silenced by small interfering RNA transfection.TM4SF1 expression in cell lines and tissues were determined by Quantitative Real-time PCR. MTS, clonogenic, Transwell assay, Flow cytometry verified cell function. By RT-PCR, Western blot, the mechanisms were studied.

Results: TM4SF1 was upregulated in both lung cancer cell lines and tissues, compared with 293 T epithelial cells. Analysis of online databases revealed that high expression of TMASF1 is associated with the older patient age, smoking habits, and poor patient survival and outcome. Knockdown of TM4SF1 substantially inhibited tumor cell growth, migration, and invasion, and enhanced the chemo-sensitivity of the lung cancer cell lines A549 and H1299 to cisplatin and paclitaxel. Furthermore, the silencing of TMASF1 induced lung cancer cell apoptosis and arrested cells at the G2/M phase. These results suggest that TM4SF1 is associated with lung cancer progression and appears to be required for tumor cell growth, maintenance of chemo-resistance and metastasis. We further found that TM4SF1 exerts these effects in part by regulating the expression of the discoidin domain receptor DDR1 and its downstream target, the Akt/ERK/mTOR pathway, and consequently alters cell sensitivity to chemo-reagents and contributes to invasion and metastasis.
\end{abstract}

Conclusions: These findings demonstrate that TM4SF1 may serve as a prognostic factor for lung cancer chemoresponse and patient outcome.

Keywords: Non-small-cell lung cancer, TM4SF1, Invasion, Migration, Apoptosis, Cell cycle, Chemo-sensitivity, DDR1, Akt, ERK

\footnotetext{
* Correspondence: cyzym@126.com; pwy16@sina.com

${ }^{4}$ Nursing College, Chongqing Medical University, Chongqing, China

${ }^{2}$ Chongqing Key Laboratory of Molecular Oncology and Epigenetics, The

First Affiliated Hospital of Chongqing Medical University, Chongqing, China

Full list of author information is available at the end of the article
}

(c) The Author(s). 2019 Open Access This article is distributed under the terms of the Creative Commons Attribution 4.0 International License (http://creativecommons.org/licenses/by/4.0/), which permits unrestricted use, distribution, and reproduction in any medium, provided you give appropriate credit to the original author(s) and the source, provide a link to the Creative Commons license, and indicate if changes were made. The Creative Commons Public Domain Dedication waiver (http://creativecommons.org/publicdomain/zero/1.0/) applies to the data made available in this article, unless otherwise stated. 


\section{Background}

Lung cancer is the most common cancer worldwide, with an incidence of $11.6 \%$ [1]. Non-small-cell lung cancer (NSCLC) constitutes 85 to $90 \%$ of lung cancers and is the most common cause of cancer death [2]. Cisplatin- or paclitaxel-based adjuvant therapy is one of the major treatment approaches among the systemic therapies for NSCLC. This method has been demonstrated to have unequivocal benefits, in terms of both increasing cure rates in adjuvant and multimodality settings in earlier stages of the disease and enhancing the quality and length of life in advanced stages of the disease [2]. However, data from more than 1300 patients enrolled in eight trials of adjuvant cisplatin-based therapy have indicated a $5 \%$ survival benefit at 5 years. These disappointing results are partially due to the chemo-resistance of NSCLC [3]. Therefore, understanding the mechanisms of how NSCLC becomes resistant to chemo-reagents is crucial.

Transmembrane-4 L-six family member-1 (TM4SF1) is a small plasma membrane glycoprotein that regulates cell motility and proliferation [4]. TM4SF1 was first discovered as an antigen for immunotherapy in lung cancer and its antibody MAb L6 showed a favorable binding properties and the encouraging clinical effect [5]. Experiments on tumor cells have previously shown TM4SF1 to be important for cell growth in liver and lung cancer [6,7], motility in lung cancer [7], invasion in pancreatic cancer [8], and metastasis of breast cancer to the lungs [9]. TM4SF1 has been reported to interact with discoidin domain receptor 1 (DDR1) in breast cancer [9] and in pancreatic cancer metastasis [10]. DDR1 is an up-stream regulator of the $A K T / m T O R$ pathway [11], a pathway involved in chemo-resistance in multiple cancers, including lung cancer [12]. Consequently, we hypothesized that TM4SF1 might also participate in the process of cancer chemo-resistance through regulating DDR1. However, the role of TM4SF1 in lung cancer chemo-sensitivity has not been investigated. We report here that TM4SF1 regulates lung cancer chemo-sensitivity and apoptosis through the $D D R 1$-activated $A K T / m T O R$ signaling pathway.

\section{Methods}

\section{Cell lines and tumor samples}

NSCLC cell lines (A549, H1299) were purchased from the ATCC (Manassas, VA,USA). Both cell lines are lung adenocarcinoma cell lines. Cell lines were cultured in RPMI 1640 as described previously [13]. Human embryonic kidney epithelial cell $293 \mathrm{~T}$ cell lines were used as a normal control. $293 \mathrm{~T}$ cells were cultured in DMEM with 10\% Fetal Bovine Serum. Fresh NSCLC tissues and tumor-adjacent tissues were obtained from patients who underwent lobectomy at the Department of Cardiothoracic Surgery (Patient clinical features were listed in Table 1) in the First Affiliated Hospital of Chongqing Medical University (Chongqing,
Table 1 Clinicopathological features of 25 NSCLC patients

\begin{tabular}{|c|c|}
\hline Clinicopathological features & Number $(n=25$ \\
\hline \multicolumn{2}{|l|}{ Gender } \\
\hline Male & 20 \\
\hline Female & 5 \\
\hline \multicolumn{2}{|l|}{ Age } \\
\hline$\leq 50$ & 5 \\
\hline$>50<60$ & 17 \\
\hline$\geq 60$ & 3 \\
\hline \multicolumn{2}{|l|}{ Phase } \\
\hline 1 & 9 \\
\hline$\|$ & 6 \\
\hline III & 7 \\
\hline IV & 3 \\
\hline \multicolumn{2}{|l|}{ Tumour size } \\
\hline$<3.0 \mathrm{~cm}$ & 9 \\
\hline$\geq 3.0 \mathrm{~cm} \leq 5.0 \mathrm{~cm}$ & 13 \\
\hline$\geq 5.0 \mathrm{~cm} \leq 7.0 \mathrm{~cm}$ & 2 \\
\hline$\geq 7.0 \mathrm{~cm}$ & 1 \\
\hline \multicolumn{2}{|l|}{ Lymph node metastasis } \\
\hline present & 8 \\
\hline absent & 17 \\
\hline \multicolumn{2}{|l|}{ Distant Metastasis } \\
\hline present & 4 \\
\hline absent & 21 \\
\hline \multicolumn{2}{|l|}{ Smoking status } \\
\hline smoker & 17 \\
\hline non-smoker & 8 \\
\hline \multicolumn{2}{|l|}{ Tumor Histology } \\
\hline Adenocarcinoma & 11 \\
\hline SqCa & 13 \\
\hline Adeno+SqCa & 1 \\
\hline
\end{tabular}

China). This research was approved by the Institutional Ethics Committees of the First Affiliated Hospital of Chongqing Medical University and followed the principles of the Declaration of Helsinki. Patient consent forms were signed by each patient who participated in this study.

\section{RNA extraction}

RNA was extracted from cell lines and tissue samples with TRIzol reagent (Molecular Research Center, Cincinnati, OH, USA) and treated with DNase I. The RNA was stored at $-80^{\circ} \mathrm{C}$, and its concentration was measured by spectrophotometry. RNA was reverse-transcribed according to the instructions of the Reverse Transcription System (Promega, Madison, WI, USA). Semi-quantitative PCR was carried out with Go-Taq DNA polymerase (Promega), and reaction conditions were as previously described [12]. 
Real-time quantitative PCR used ABI SYBR Green on an ABI 7500 Real-Time PCR Detection System (Applied Biosystems, Foster City, CA, USA). GAPDH was used as a loading control.

The primers designed for human TM4SF1 were as follows: forward, 5'-GGTTCTTTTCTGGCATCGTAGGAGGTG-3';reverse, 5' -CTGGCCGAGGGAATCAAGACATAGTG-3'. The primer for GAPDH was as follows: forward,5'-GGAGTCAACGGATTTGGT-3'; reverse, 5'-GTGATGGGATTTCCATTGAT-3'.

\section{Knockdown of TM4SF1 with small interfering RNAs (siRNAs)}

TM4SF1-target-specific siRNA, negative-control TM4 SF1-target-specific siRNA and negative-control siRNA (NC) were all synthesized by OriGene, (Rockville, MD,USA), Three pairs of siRNAs that targeted TM4SF1 were designed and synthesized: siRNA-TM4SF1-A: 5' GCGAUGCUUUCUUCUGUAUTT-3' (forward), 5' -AU ACAGAAGAAAGCAUCGCTT-3' (reverse); siRNA-TM 4SF1-B:5'-GGCUCUUGGUGGAAUUGAATT-3' (forward), 5'-UUCAAUUCCACCAAGAGCCTT-31 (reverse); siRNA-TM4SF1-C: 5'-GCUCUCACCAACA GCAAUATT-3' (forward),5' -UAUUGCUGUUGGUGAGAGCTT-3'(reverse). The sequence of NC-siRNA was as follows: 5'-UUCUCCGAACGUGUCACGUTT-3' (forward); 5' -ACGUGACACGUUCGGAGAATT-3' (reverse). Transfections were performed using Lipofectamine-2000 transfection system as described previously [8].

\section{Colony-formation assays}

A549 and H1299 cells transfected with siRNA or NC were plated in six-well plates at a density of 1200 cells/ well in culture medium $24 \mathrm{~h}$ after transfection medium was changed every 3 daysAfter $14 \mathrm{~d}$, the cells were fixed with 4\% PFA and stained with crystal violet solution. Colonies with $>50$ cells per colony were counted.

\section{Cell proliferation assays}

Cell proliferation was assessed with MTS assays (Abcam, Cambridge, MA,USA) and PCNA (Proliferating Cell Nuclear Antigen) expression. PCNA is known as a marker of cell proliferation besides Ki67, testing PCNA expression could further understand the effects of TM4SF1 on NSCLC cell proliferation. For MTS, NCand siRNA-transfected cells were seeded in 96-well plates (3000 cells/well) with $100 \mu \mathrm{L}$ of medium. Cells were incubated for 24,48 or $72 \mathrm{~h}$, and then $100 \mu \mathrm{L} /$ well serum-free medium containing $20 \mu \mathrm{L}$ MTS reagent was added to each well. Plates were then incubated at $37^{\circ} \mathrm{C}$ for an additional $2 \mathrm{~h}$. Absorbance was measured at 490 $\mathrm{nm}$ with a microplate reader (Multiskan MK3; Thermo Fisher Scientific,). PCNA expression was carried out by western blot.

\section{Chemo-reagent treatment}

Cisplatin and paclitaxel were purchased from QiLu Pharma (Shan Dong, China). TM4SF1 knockdown cells and NC cells were treated with cisplatin at concentrations of $0,2.5,5,10,15$, or $20 \mu \mathrm{g} / \mathrm{ml}$ for $12 \mathrm{~h}$, or with paclitaxel at concentrations of $0,2.5,5,10,20$, or $40 \mathrm{ng} / \mathrm{ml}$ at $24 \mathrm{~h}$ and then collected for MTS analyses to quantify cell viability of TM4SF1 knockdown cells and NC cells.

\section{Cell migration and invasion assays}

Cell migration and invasion ability were measured with Transwell chambers $(8-\mu \mathrm{m}$ pore size; Corning, NY, USA), as described previously [14]. In general, $4 \times 10^{4}$ cells/well diluted in $100 \mu \mathrm{l}$ serum-free medium was in the upper transwell filter inserts in 24-well plates. The lower chambers contained $800 \mu \mathrm{l}$ medium containing $10 \%$ FBS. Cells were incubated at $37{ }^{\circ} \mathrm{C}$ in a $5 \% \mathrm{CO}_{2}$ incubator for $24 \mathrm{~h}$. For invasion assay, the upper chamber was pre-coated with 1:5 diluted Matrigel ${ }^{\mathrm{TM}}$.

\section{Flow cytometry analysis of the cell cycle and apoptosis}

For apoptosis, $1 \times 10^{6} \mathrm{~A} 549$ and $\mathrm{H} 1299$ cells were transiently transfected with siRNA or NC, follow the manufacture's instruction of Lipofectamine-2000 transfection system, then analyzed with Annexin V-FITC/PI $\left(5 \mathrm{ul} / 1 \times 10^{6}\right.$ cell $)$ following the manufacture's protocol (BD Medical Technology) staining for apoptosis analysis. Data were analyzed with CellQuest $^{\mathrm{Tm}}$ Pro (BD Biosciences, San Jose, CA, USA).

For cell cycle analysis, cells were collected as before. Cells were then sorted with a FACSCalibur machine (BD Biosciences, Franklin Lakes, NJ, USA). Data were analyzed in ModFit 3.0 software (Verity Software House, Topsham, ME, USA).

\section{Western blotting}

SiRNA- TM4SF1or NC-transfected cells $\left(1 \times 10^{6}\right)$ were washed three times with ice-cold PBS and then lysed in lysis buffer (4\%v SDS, 20\% glycerol,120 mM Tris-HCI, $\mathrm{PH}$ 6.8). Then, $40 \mu \mathrm{g}$ protein samples were separated by SDS-PAGE, transferred to polyvinylidene difluoride membranes and immune-stained as previously described [15]. Signals were detected with an ECL Plus Western Blotting Detection Reagents (RPN2132, GE HealthcareLife Sciences, UK). Primary antibodies to the following targets were used: TM4SF1 (sc-103,267) and p-ERK (sc-7383), both Santa Cruz Biotechnology, CA, USA, cleaved caspase-3 (\#9661), cleaved caspase-7 (\#9491, cleaved caspase-9 (\#9504), cleaved PARP (\#9661), DDR1 (\#3917), p-mTOR (\#5536, Cell Signaling, MA), and p-AKT (\#4060), all Cell Signaling Technology, Danvers, MA, USA, with GAPDH (bsm-51,010 M; BIOSS, China), as a loading control. The dilution of primary and secondary antibodies was used according to the company's recommendations, Primary antibodies were diluted in 
1:1000, secondary antibodies were diluted in 1:3000. The bands were detected with an enhanced chemiluminescence kit (Amersham Pharmacia Biotech, Piscataway, NJ, USA).

\section{mTOR inhibitor treatment}

TM4SF1 knocking down H1299 cell lines and NC cells were treated with mTOR inhibitor Dactolisib at $50 \mu \mathrm{M}$ for $24 \mathrm{~h}$ [16]. Protein was then extracted and western blotting was used as described before.

\section{Analyses using online databases}

The Human Protein Atlas (https://www.proteinatlas.org/) were used to analyze the correlation between TM4SF1 expression and patient survival in NSCLC. The relationships between TM4SF1 expression and NSCLC patient clinical signatures were analyzed with the UALCAN database (ualcan.path.uab.edu/). The threshold search value used for this study was a $p$-value $<0.05$.

\section{Statistical analysis}

All data are representative of three independent experiments and are presented as mean \pm SD. SPSS16.0 software was used for statistical analyses. For all tests, $p<0.05$ was considered statistically significant.

\section{Results}

TM4SF1 is over-expressed in lung cancer cell lines and lung cancer tissue samples

We sought to understand the biological function of TM4SF1 in lung cancer, specifically whether it promotes or suppresses lung cancer development. We first evaluated its expression in both lung cancer cell lines and tissue samples. With RT-PCR, we identified that TM4SF1 was up-regulated in the lung cancer cell lines A549, H1299, H1650, H460, H446, and H1466, compared with the epithelial cell $293 \mathrm{~T}$ cells (Fig. 1d upper panel), suggesting that it may potentially promote lung cancer. Furthermore, through real-time quantitative PCR, we quantified TM4SF1 expression in 25 paired lung cancer tissue and its related adjacent non-tumor tissues. TM4SF1 was over-expressed in the lung cancer tissues relative to the adjacent non-tumor tissues in 21 out of the 25 pairs $(84 \%)$, suggesting that it may be an oncogene in lung cancer (Fig. 1c,e). RT-PCR was further used to confirm the TM4SF1 expression in five pairs of lung tumor tissues and non-tumor tissues (Fig. 1d lower panel). These findings together demonstrated that TM4SF1 is up-regulated in both lung cancer cell lines and tumor tissues, and it may be a potentially related to lung cancer progression.
TM4SF1 expression is related to patient clinicopathological features and outcomes

To assess any correlation between TM4SF1 gene expression and patient clinicopathological features in NSCLC samples, we used the UALCAN database (www.ualcan. path.uab.edu/). The results showed that in lung squamous cell cancer, which accounts for most (80\%) cases of lung cancer, high TM4SF1 expression was associated with older patient age, in addition, its high expression was related to patient smoking status (Fig. 1b), with TM4SF1 being expressed more highly in former smokers than in non-smokers .

We further analyzed the relationship between TM4SF1 expression and NSCLC patient survival the Human Protein Atlas database (www.proteinatlas.org/ ). The $\mathrm{Hu}$ man Protein Atlas database confirmed in both lung adenocarcinoma $(p=0.0017)$ and lung cancers, including both squamous cell cancer and adenocarcinoma ( $p$ $=0.00274)$, high TM4SF1 expression was associated with poor patient survival)(Fig. 1a). These results indicated that TM4SF1 expression may serve as an early biomarker to predict the prognosis of lung cancer patients.

Together, these findings indicate that high TM4SF1 expression, an age over 80, a former smoking habit are risk factors for poor patient outcome in lung cancer. TM4SF1 may serve as a biomarker of lung cancer outcome and prognosis.

\section{Down-regulation of TM4SF1 in A549 and H1299 cells suppresses cell growth, migration, and invasion}

To investigate the function of TM4SF1 in lung cancer cells, we transiently transfected A549 and H1299 cells with NC or siRNA-TM4SF1 and confirmed the knockdown of TM4SF1 by RT-PCR and western blotting. The results showed that both the TM4SF1 mRNA and protein levels were significantly decreased by siRNA-TM4SF1 (Fig. 2a). We then analyzed the effects of TM4SF1 silencing on lung cancer cell proliferation, migration and invasion in vitro. By colony formation assay, we found the colonies formed in the TM4SF1-silenced group being smaller and fewer than those in the control group $(p<0.001)$ (Fig. 2 b). In Transwell chamber assays, silencing of TM4SF1 markedly decreased the migration and invasion of lung cancer cells compared with wild-type and negative control cells ( $p<0.001)$, (Fig. 4c). Moreover, wound healing assays revealed that scratches in TM4SF1-silenced A549 and H1299 cell lines healed significantly more slowly than those in negative control and wild-type cells $24 \mathrm{~h}$ after wounding $(p<0.001)$. The wound healing assays also indicated that knockdown of TM4SF1 significantly inhibited lung cancer cell migration ability (Fig. 3a). With MTS, we analyzed the silencing of TM4SF1 on cell viability and cell growth in lung cancer cell lines (Fig. 3b), by western blot,the PCNA 

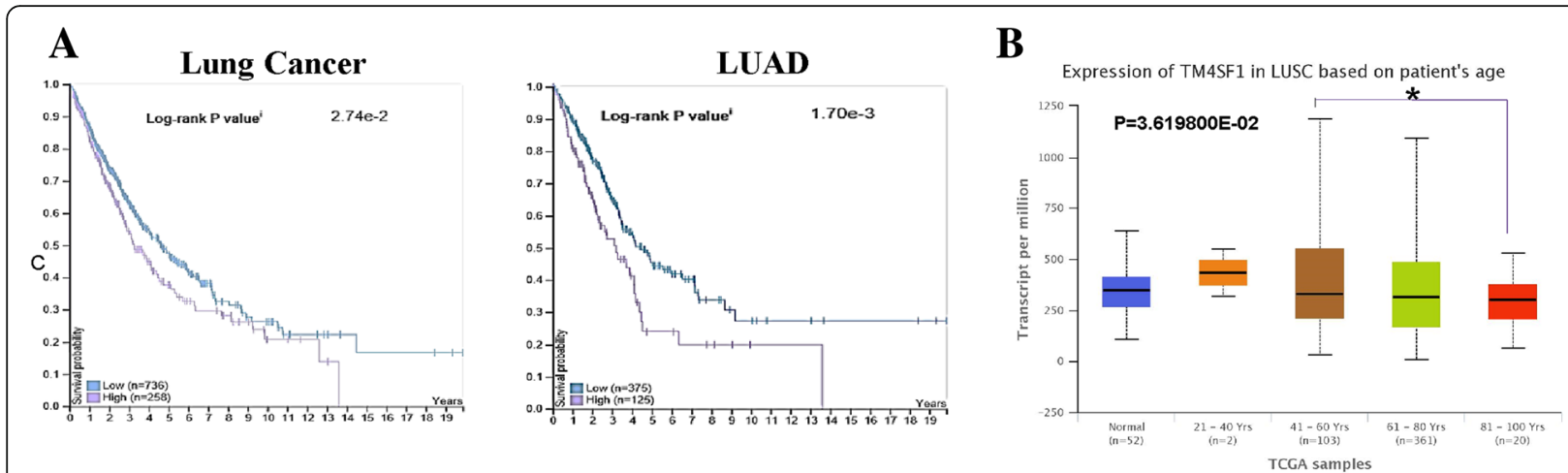

C

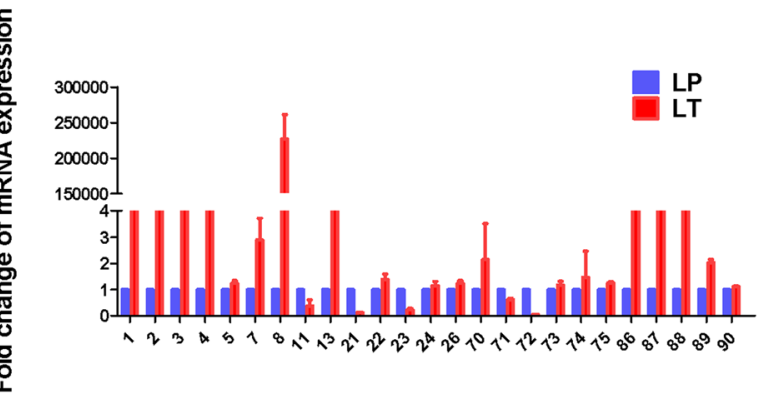

D
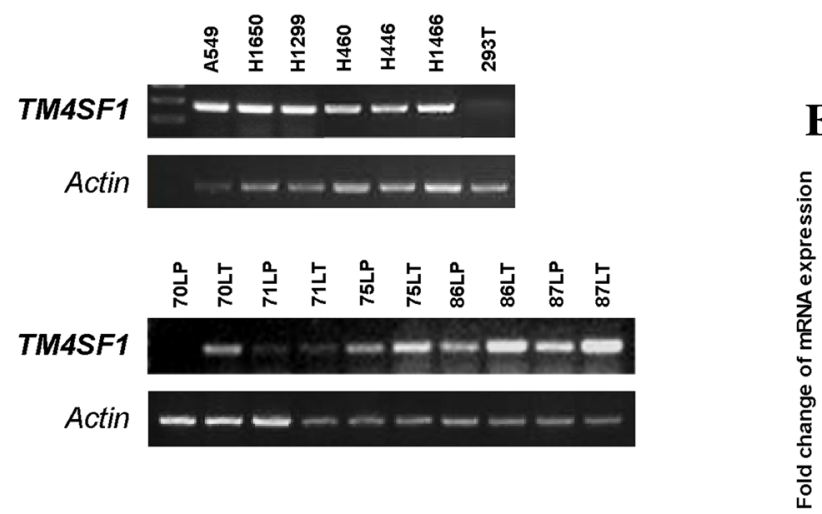

Expression of TM4SF1 in LUSC based on patient's smoking habits

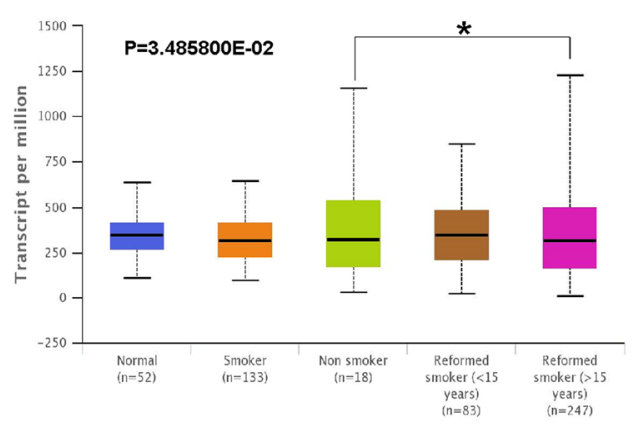

$\mathbf{E}$

TCGA samples

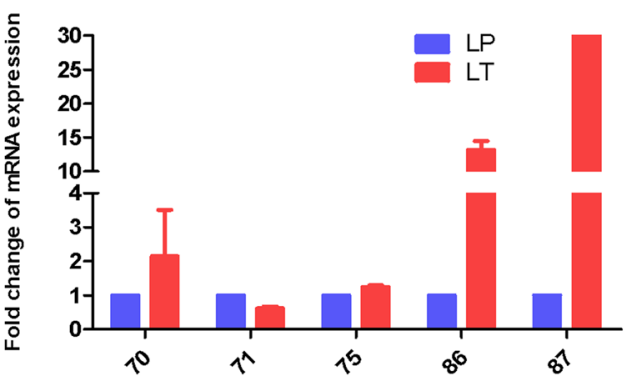

Fig. 1 Over-expression of TMASF1 in NSCLC and it was associated with patients' pathological features. a The high expression of TM4SF1 expression in NSCLC was associated with poor patient survival, data from The Human Protein Atlas (https://www.proteinatlas.org/). b The high expression of TM4SF1 was related to patient age and smoking habits, data from UALCAN database (ualcan.path.uab.edu/).c The expression of TM4SF1 in NSCLC tissues and non-tumor adjacent surgical margine by q-PCR. $\mathbf{d}$ RT-PCR demonstrated TM4SF1 expression in NSCLC cell lines and tissue samples. e q-PCR verified the RT-PCR results of TM4SF1 expression in RT-PCR

expression was downregulated by knocking down TM4SF1(Fig.4 d) . Compared with the negative control, knockdown of TM4SF1 significantly reduced cell viability $(p<0.001)$. Together, these findings suggested that TM4SF1 inhibits lung cancer cell migration and invasion ability.

\section{TM4SF1-induced cell cycle arrest of NSCLC cells at the G2/} $M$ phase

Cell cycle regulation is one reason among the complex mechanisms of NSCLC chemo-resistance. To further test whether TM4SF1 plays a role in cell cycle regulation, we performed flow cytometry cell cycle distribution assays. In the siRNA-TM4SF1 transfected group, we found that the percentage of cells in the G2/M phase was greater than that in the control group (Fig. 4a), and the cell population in the $S$ phase was also greater. These findings strongly suggested that the silencing of TM4SF1 regulates cell cycle arrest at the $\mathrm{G} 2 / \mathrm{M}$ phase.

\section{Silencing of TM4SF1 enhances the chemo-sensitivity of NSCLC cells to paclitaxel and cisplatin}

Because TM4SF1 expression led to G2/M cell cycle arrest, we investigated the effect of TM4SF1 knockdown on the 


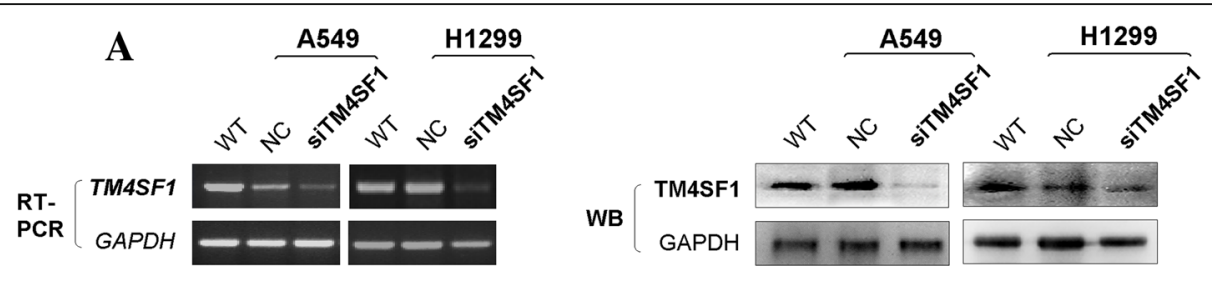

B
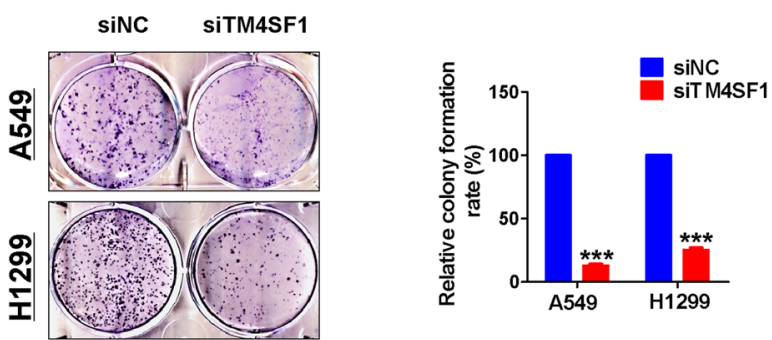

$\mathbf{C}$
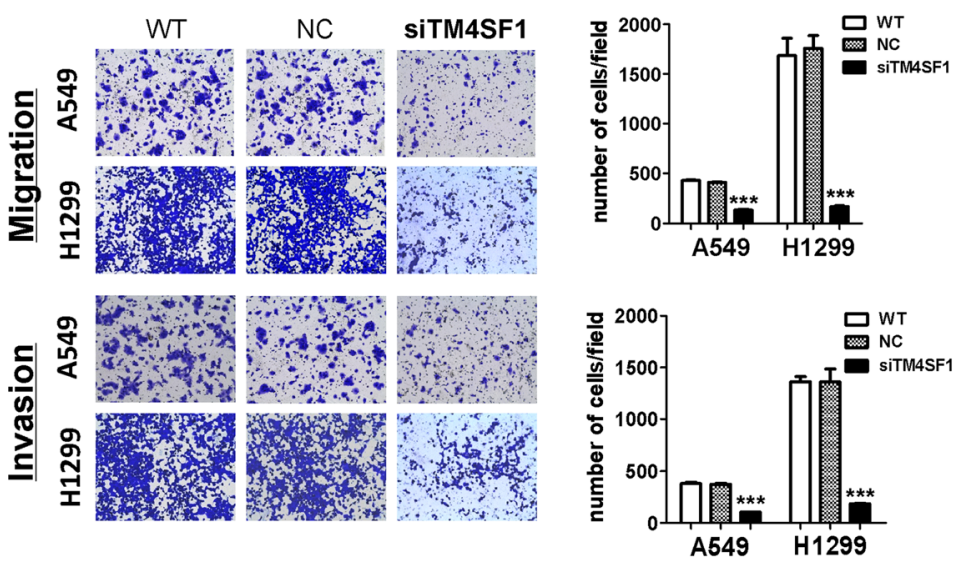

Fig. 2 The knockdown of TM4SF1 suppressed colony formation and cell invasion and migration in A549 and H1299 cell lines. a RT-PCR and western blots confirmed the silencing of TM4SF1 in A549 and H1299 cell lines. b The silencing of TM4SF1 by siRNA inhibited colony formation in A549 and H1299 cell lines (left panel). Right panel showed quantitative analysis of colony formation. The numbers of colonies in NC cells were set to $100 \%$. Values are expressed as the mean \pm SD from three independent experiments, and the asterisk indicates the statistical significance compared to the controls (***, $p<0.001)$. c Cell migration and invasion assay in NC and TM4SF1 silenced A549 and H1299 cell lines by a $24-$ transwell system. Original magnification $\times 10$, Asterisks indicate a significant level of proliferation compared with controls $\left({ }^{* * *}, p<0.001\right)$, the right panel indicates the quantitative analysis of transwell cell invasion and migration assay

chemo-sensitivity of NSCLC cells to cell cycle-phase-specific and phase-nonspecific chemotherapeutic drugs. Paclitaxel and cisplatin are the most commonly used chemo-therapy reagents for NSCLC. Paclitaxel functions by inducing cell G2/M arrest [14], while cisplatin is known as a cell cycle-phase-nonspecific chemo-reagent [12]. As shown in Fig. 4b, knocking down TM4SF1 with siRNA increased the sensitivity of A549 and H1299 cells to paclitaxel and cisplatin, as compared with the controls. The IC50 of cisplatin in A549 /SiRNA-TM4SF1 cells was $5.28 \mu \mathrm{g} / \mathrm{ml}$ vs $15.27 \mu \mathrm{g} / \mathrm{ml}$ in control cells; in H1299/SiRNA-TM4SF1 cells, the IC50 was $9.73 \mu \mathrm{g} / \mathrm{ml}$ vs $28.21 \mu \mathrm{g} / \mathrm{ml}$ in NC H1299 cells $(p<0.001)$. In A549 cell line, the IC50 of paclitaxel was reduced by half by the silencing of TMASF1. These results indicated that TM4SF1 plays a role in the NSCLC chemo-resistance.
TM4SF1 induces apoptosis in NSCLC cells

The influence of TM4SF1 on tumor cell proliferation suggested that it may induce cell apoptosis resistance in NSCLC cell lines. Flow cytometry of A549 and H1299 cells confirmed that, compared with control treatment, silencing of TM4SF1 induced cell apoptosis in both cell lines $(p<0.01$ in A549 cells and $p<0.001$ in H1299 cells),(Fig. 4c). These results indicate that TM4SF1 plays a part in NSCLC cell resistance to apoptosis and thus may be related to NSCLC chemo-resistance.

\section{TM4SF1 enhances NSCLC chemo-sensitivity through induction of apoptosis and the DDR1/AKT signaling pathway}

To further explore the mechanism underlying the ability of TM4SF1 to induce NSCLC sensitivity to chemo-therapy, 

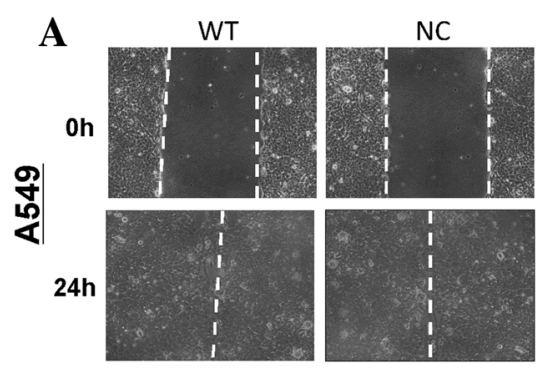
SITM4SF1
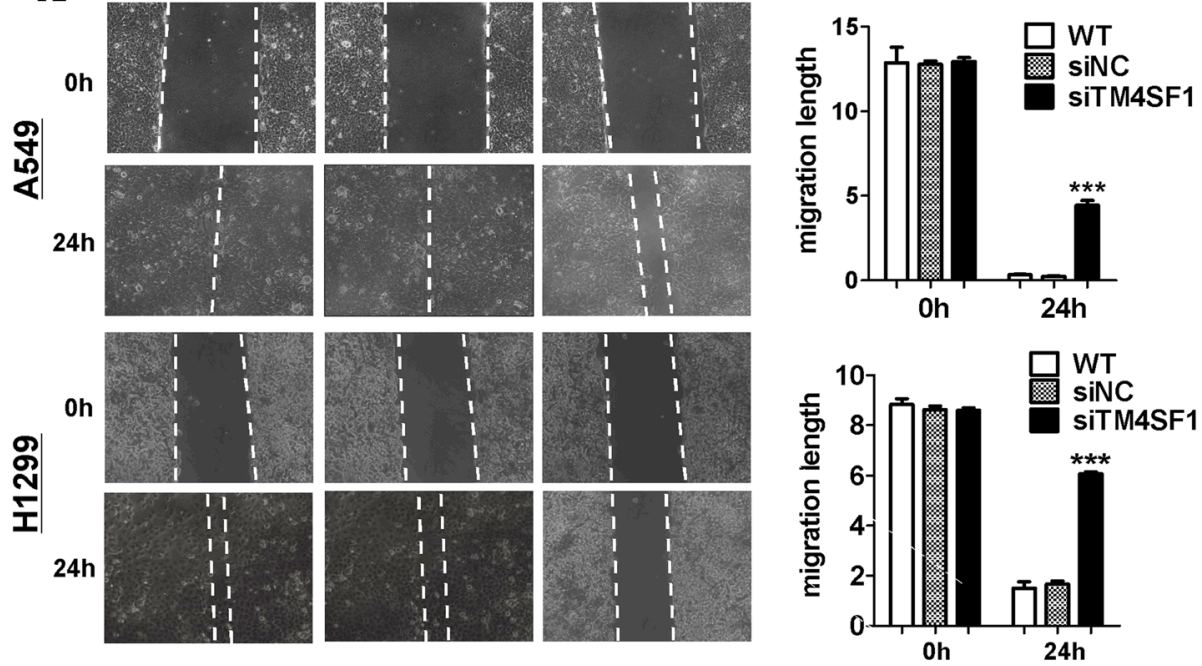

B
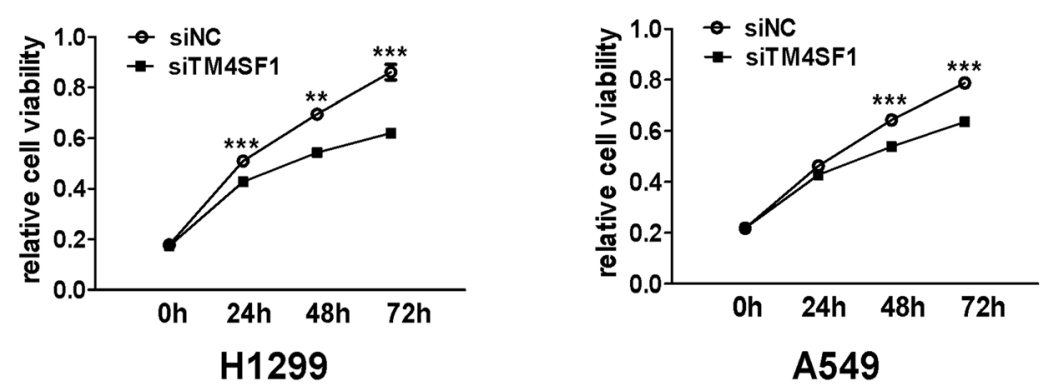

Fig. 3 The knockdown of TMASF1 suppressed cell wound healing ability and cell proliferation in A549 and H1299 cell lines. a Representative image (left) of wound healing assay in NC and TM4SF1 knocking down A549 and H1299 cells. The distance of cell migration was collected to make the statistical figure (right). $\mathbf{b}$ The ability of cell proliferation was measured in NC and TM4SF1 knocking cells by MTS. And the asterisk indicates the statistical significance compared to the controls $\left(* * *, p<0.001,{ }^{* *}, p<0.01\right)$

we used western blotting to verify the effects of TM4SF1 on apoptotic markers and possible signaling pathways in NSCLC. TM4SF1 knockdown significantly induced PARP (poly (ADP-ribose) polymerase) cleavage and up-regulated caspases 7,9 , and 3 , suggesting that TM1SF4 promotes lung cancer cell apoptosis and may induce lung cancer cell resistance to chemo-therapy-induced apoptosis, and play a part in lung cancer chemo-resistance. Furthermore, DDR1 has been reported to interact with TM4SF1 and to induce tumor metastasis. Through western blotting, we verified that TM4SF1 also regulated DDR1 and the phosphorylation of its downstream targets $A K T, E R K$, and $m T O R$ (Fig. 4e). To further identify whether TM4SF1 regulated DDR1/AKT/mTOR pathway, mTOR inhibitor Dactolisib was used, Knocking down of TM4SF1 can further down-regulated DDR1/AKT pathway after inhibitor treatment. (Fig. 4d) These results suggested that, through DDR1, TM4SF1 regulates the AKT/ERK pathway and is involved in NSCLC chemo-resistance.

\section{Discussion}

TM4SF1 is an integral membrane glycoprotein that can transport extracellular signals to the cytoplasm. Originally identified as a tumor-specific antigen, TM4SF1 has been reported to be over-expressed in many epithelial cancers, such as pancreatic cancer [7], prostate cancer [17], liver cancer [5], and especially lung cancer [18]. Additionally, TM4SF1 has been reported to be an oncogene promoting tumor cell invasion metastasis, tumor angiogenesis, and the progression of multiple cancers [15].

Chemo-resistance is the key mechanism of the unfavorable outcome of lung cancer. Limited efficacy, significant toxicity, and the poor initial performance status of chemo-therapy was reported in advanced lung cancer [19] .Finding a biomarker that cloud be an early predict marker that can predict lung cancer patients' response to chemo-therapy is in urgent need.

In the present study, we examined the function of TM4SF1 in NSCLC, particularly its role in NSCLC 
A
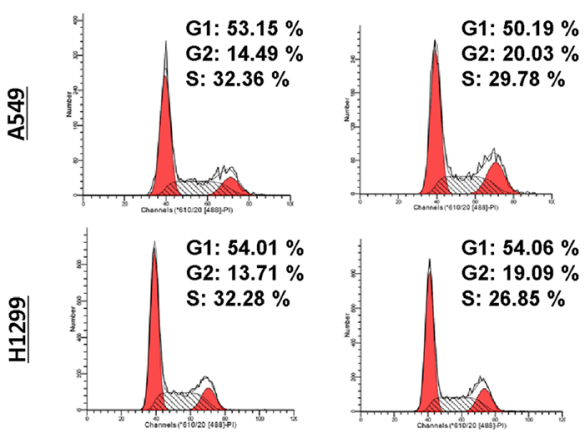

B

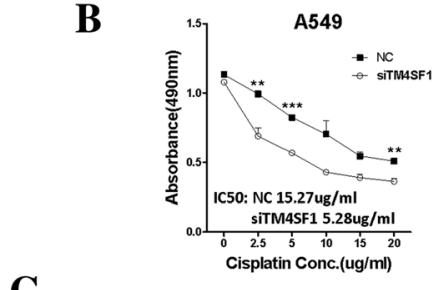

C
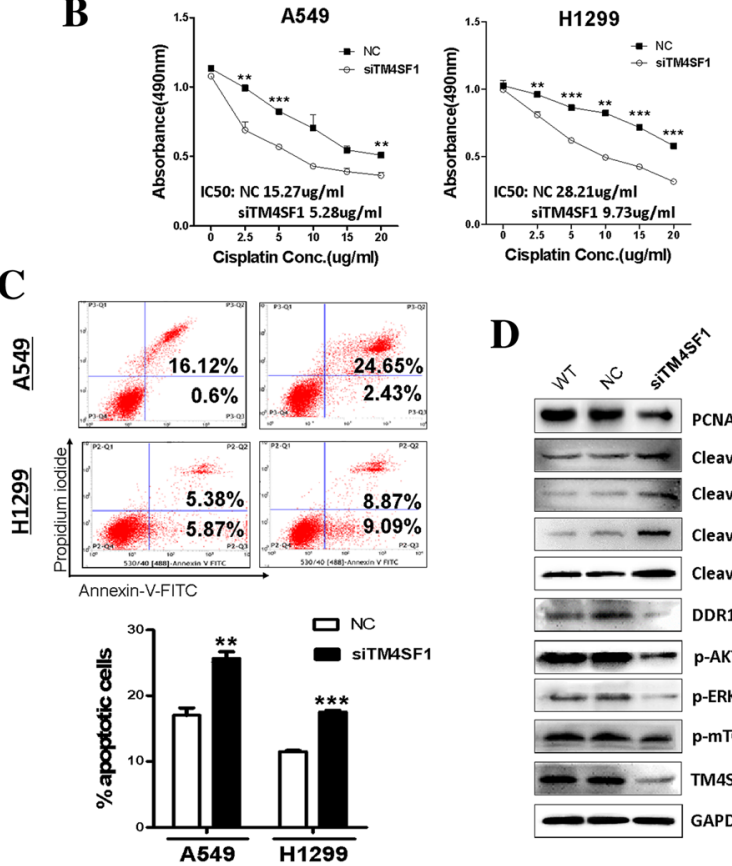

D

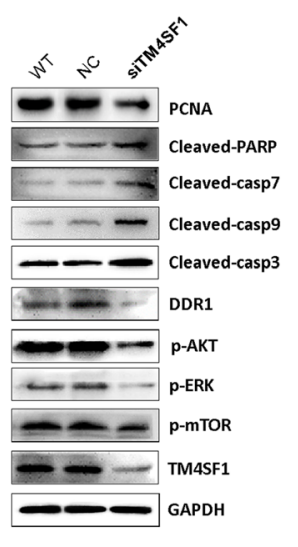

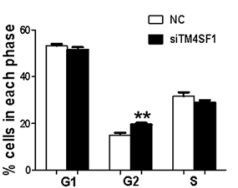
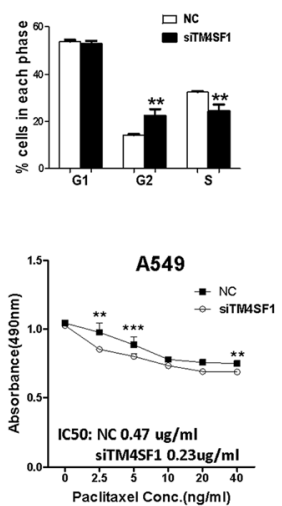

$\mathbf{E}$

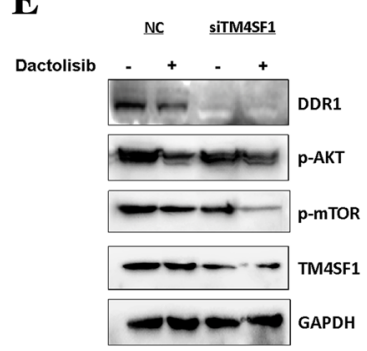

Fig. 4 The silencing of TM4SF1 induces cell cycle G2/M arrest, apoptosis and affects DDR1 signaling and apoptotic markers in NSCLC cell lines. a Representative distribution of A549 and H1299 cell lines both in NC and TM4SF1 silencing cells (left); The distribution and percentage of cells in the G1, S and G2/M phases of the cell cycle are indicated (right).b Knocking down of TM4SF1 sensitized NSCLC cells to cisplatin and paclitaxel, The cell viability was detected by MTS assay after cells were treated with chemotherapy drugs paclitaxel for $24 \mathrm{~h}$ or cisplatin for $12 \mathrm{~h}$. All experiments were performed in triplicate, respectively. ${ }^{*} p<0.05,{ }^{* *} p<0.01,{ }^{* *} p<0.001$. c Induction of apoptosis detected by flow cytometric analysis with Annexin V-FITC and PI-staining (right). Quantitative analysis of apoptosis (**p $<0.01,{ }^{* * *} p<0.001$ ). d Expression levels of Casp7, 9, 3, cleaved-PARP were evaluated by western blot in NC and TM4SF1 silencing cells. And the impact of silencing TM4SF1 on the expression of DDR1 and the phosphorylation of its downstream targets AKT, ERK and mTOR. e Inhibitor down-regulated DDR1, mTOR and AKT and Knocking down TM4SF1 can further lower the expression of this pathway after inhibitor treatment

chemo-resistance. First, we examined TM4SF1 expression in NSCLC cell lines and paired lung cancer tissue and adjacent non-cancerous tissue, by using RT-PCR and semi-quantitative PCR, respectively. TM4SF1 was up-regulated in both lung cancer cell lines and tissues, thus suggesting that it may be a potential oncogene in NSCLC. Then, through analysis of online databases, we found that TM4SF1 is related to important clinicopathological features of NSCLC. Online data suggested its high expression was found to be associated with the older people, and smokers. In addition, high expression of TM4SF1 was associated with poor patient survival and outcome. Thus, the expression of TM4SF1 may be a potential biomarker for early determination of lung cancer patient outcomes and treatment responses. Through in vitro cell function experiments, we verified that the silencing of TM4SF1 with siRNA inhibited proliferation of the NSCLC cell lines A549 and H1299, as well as invasion and migration, thus confirming that TM4SF1 has an oncogenic function in NSCLC. Interestingly, through MTS, we found that TM4SF1 knockdown enhanced the chemo-sensitivity of NSCLC cell lines to cisplatin and paclitaxel. Cisplatin and paclitaxel are the most commonly used and key reagents in NSCLC chemo-therapy. 
Cisplatin is known as a cell cycle-non-specific chemo-therapy drug, whereas paclitaxel induces G2/M arrest. TM4SF1 promoted chemo-resistance to both reagents in NSCLC, thus suggesting that it regulates cancer chemo-resistance through inducing cell cycle arrest and other underlying mechanisms beyond cell cycle regulation, such as inducing cell apoptosis resistance and interacting with a signaling pathway strongly involved in tumor chemo-resistance. To demonstrate the above hypothesis regarding the mechanism by which TM4SF1 regulates NSCLC chemo-sensitivity, we used flow cytometry and found that TM4SF1 silencing in A549 and H1299 cell lines induced apoptosis and cell cycle arrest at the G2/M phase. This result verified that inducing tumor resistance to apoptosis and regulating the cell cycle are the mechanisms underlying the promotion by TM4SF1 of NSCLC chemo-resistance. Secondly, we explored the possible signaling pathway involved in this chemo-resistance effect by using western blotting. DDR1 belongs to the large family of receptor tyrosine kinases based on the presence of a catalytic kinase domain with a distinct extracellular Discoidin (DS) homology domain [20]. TM4SF1 has been reported to couple with the collagen receptor tyrosine kinase DDR1 in breast cancer progression [9] and pancreatic cancer invasion [10]. DDR1 in turn interacts with MAPKs $[16,21]$ and is the up-stream regulator of the AKT pathway $[11,22]$. Both pathways have been reported to interact with each other [23] and to be highly involved in tumor chemo-resistance [24]. With MAPKs and is an up-stream regulator of the AKT pathway. Therefore, by interacting with DDR1, TM4SF1 may regulate key genes in both the MAPK and AKT pathways, thus inducing NSCLC chemo-resistance. As verified by western blotting, TM4SF1 knockdown resulted in the down-regulation of DDR1 and consequently inhibited the phosphorylation of Akt and ERK. mTOR is involved in both pathways, and its phosphorylation was also disrupted. Thus, through regulating the DDR1/ERK/Akt-mTOR pathway, TM4SF1 induces NSCLC chemo-resistance. TM4SF1 may therefore serve as a potential biomarker for predicting the treatment response in lung cancer chemo-therapy.

In general, most previous studies have focused on the role of TM4SF1 in regulating tumor invasion and metastasis. The present study demonstrated the multiple functions of TM4SF1 in NSCLC, especially its regulation of chemo-resistance and its role in regulating DDR1/ERK/ Akt-mTOR pathway. These results provide another direction for the study of TM4SF1 in cancer development and demonstrate the potential value of TM4SF1 in predicting lung cancer chemo-response.

\section{Conclusions}

Our findings suggest that TM4SF1 is an oncogene in NSCLC. Overexpression of TM4SF1 is a common event in NSCLC. The expression of TM4SF1 was closely related to patient age, smoking habits and poor survival time,so detecting the expression level of TM4SF1 may be a potential approach in the early determination of prognosis and treatment response of NSCLC. Through in vitro cell function analyze, we demonstrated that the silencing of TM4SF1 inhibited NSCLC cell proliferation, invasion, migration and especially enhanced lung cancer cells chemo-sensitivity to cisplatin and paclitaxel. Interestingly, by western blot, we verified that interacting with DDR1 and its downstream target ERK/Akt-mTOR is the possible mechanism of TM4SF1 enhanced NSCLC chemo-sensitivity.

However, more patients and a longer follow-up time are needed to verify the correlation between TM4SF1 expression and patient survival, treatment response and other clinical aspects. This will provide more detailed information of the role of TM4SF1 in NSCLC early diagnosis, precision classification and treatment. Furthermore, in the exploration of the molecular mechanism underlying the oncogenic function of TM4SF1, more studies will be needed to find more direct evidences that TM4SF1 directly regulates $D D R 1$ and might be involved in the network of $D D R 1$ signaling pathways.

\section{Abbreviations}

Akt: Protein kinase B (PKB); DDR1: discoidin domain receptor 1 ;

ERK: Extracellular signal-regulated kinase; MAPK: Mitogen-activated protein kinase; mRNA: Messenger Ribonucleic Acid; mTOR: Mechanistic target of rapamycin; MTS: (3-(4,5dimethylthiazol-2-yl)-5-(3-carboxymethoxyphenyl)-2-(4sulfophenyl)-2H-tetrazolium); NSCLC: Non-small cell lung cancer; PARP: Poly ADP-ribose polymerase; PI3K: Phosphoinositide 3-kinase;

TM4SF1: Transmembrane-4 L-six family member-1

\section{Funding}

This work is supported by Major project of Chongqing Municipal Health Commission \#2016ZDXM006 and Science Commission of Yuzhong District, Chongqing, China \#2017-18.

Availability of data and materials

The authors will make readily reproducible materials described in the manuscript, including software, databases and all relevant raw data, freely available to scientists.

\section{Authors' contributions}

LY carried out most of the experiment and writing this manuscript; CP did the RT-PCR; JT carried out cell function tests; YW and CW helped the mechanism exprements; ZQ helped in the bioinformation analysis; TX helped the design and all through the research, provided siRNAs and designed the primers; YZ and WP supported the whole study. All authors read and approved the final manuscript.

Ethics approval and consent to participate

Use of archived human lung tissue was approved by the Ethics Committees of the First Affiliated Hospital of Chongqing Medical University.

Consent for publication

We have obtained consent to publish from the participant.

Competing interests

The authors declare that they have no competing interests. 


\section{Publisher's Note}

Springer Nature remains neutral with regard to jurisdictional claims in published maps and institutional affiliations.

\section{Author details}

'Department of Cardiothoracic Surgery, The First Affiliated Hospital of Chongqing Medical University, Chongqing, China. ${ }^{2}$ Chongqing Key Laboratory of Molecular Oncology and Epigenetics, The First Affiliated Hospital of Chongqing Medical University, Chongqing, China. ${ }^{3}$ Department of Oncology, Chongqing University Cancer Hospital\& Chongqing Cancer Institute, Chongqing, China. ${ }^{4}$ Nursing College, Chongqing Medical University, Chongqing, China.

Received: 29 November 2018 Accepted: 13 May 2019

Published online: 29 May 2019

\section{References}

1. Bray F, et al. Global cancer statistics 2018: GLOBOCAN estimates of incidence and mortality worldwide for 36 cancers in 185 countries. CA Cancer J Clin. 2018.

2. Wakelee H, Kelly K, Edelman MJ. 50 years of progress in the systemic therapy of non-small cell lung cancer. Am Soc Clin Oncol Educ Book. 2014:177-89.

3. Keller SM, et al. A randomized trial of postoperative adjuvant therapy in patients with completely resected stage II or IIIA non-small-cell lung cancer. Eastern cooperative oncology group. N Engl J Med. 2000;343(17):1217-22.

4. Cao R, et al. TM4SF1 regulates apoptosis, cell cycle and ROS metabolism via the PPARgamma-SIRT1 feedback loop in human bladder cancer cells. Cancer Lett. 2018;414:278-93.

5. Goodman GE, et al. Phase I trial of murine monoclonal antibody L6 in breast, colon, ovarian, and lung cancer. J Clin Oncol. 1990;8(6):1083-92.

6. Huang YK, Fan XG, Qiu F. TM4SF1 promotes proliferation, invasion, and metastasis in human liver Cancer cells. Int J Mol Sci. 2016;17(5).

7. Chang YW, et al. CD13 (aminopeptidase N) can associate with tumorassociated antigen $\mathrm{L} 6$ and enhance the motility of human lung cancer cells. Int J Cancer. 2005;116(2):243-52.

8. Cao J, et al. TM4SF1 regulates pancreatic Cancer migration and invasion in vitro and in vivo. Cell Physiol Biochem. 2016;39(2):740-50.

9. Gao H, et al. Multi-organ site metastatic reactivation mediated by noncanonical Discoidin domain receptor 1 signaling. Cell. 2016;166(1):47-62.

10. Yang JC, et al. TM4SF1 promotes metastasis of pancreatic Cancer via regulating the expression of DDR1. Sci Rep. 2017;7:45895.

11. Ongusaha PP, et al. p53 induction and activation of DDR1 kinase counteract p53-mediated apoptosis and influence p53 regulation through a positive feedback loop. EMBO J. 2003;22(6):1289-301.

12. Chen TJ, et al. NBM-T-BMX-OSO1, an Osthole derivative, sensitizes human lung Cancer A549 cells to cisplatin through AMPK-dependent inhibition of ERK and Akt pathway. Cell Physiol Biochem. 2015;36(3):893-906.

13. Ye $L$, et al. The $19 q 13$ KRAB zinc-finger protein ZFP82 suppresses the growth and invasion of esophageal carcinoma cells through inhibiting NFkappaB transcription and inducing apoptosis. Epigenomics. 2018.

14. Zhang $Y$, et al. The new $6 q 27$ tumor suppressor DACT2, frequently silenced by CpG methylation, sensitizes nasopharyngeal cancer cells to paclitaxel and 5-FU toxicity via beta-catenin/Cdc25c signaling and G2/M arrest. Clin Epigenetics. 2018;10(1):26.

15. Lin Cl, et al. TM4SF1: a new vascular therapeutic target in cancer. Angiogenesis. 2014;17(4):897-907.

16. El Azreq MA, et al. Discoidin domain receptor 1 promotes Th17 cell migration by activating the RhoA/ROCK/MAPK/ERK signaling pathway. Oncotarget. 2016;7(29):44975-90.

17. Allioli N, et al. TM4SF1, a novel primary androgen receptor target gene over-expressed in human prostate cancer and involved in cell migration. Prostate. 2011;71(11):1239-50.

18. Kao YR, et al. Tumor-associated antigen L6 and the invasion of human lung cancer cells. Clin Cancer Res. 2003;9(7):2807-16.

19. Lewis J, et al. Before or after: evolving neoadjuvant approaches to locally advanced non-small cell lung Cancer. Front Oncol. 2018;8:5.

20. Rammal H, et al. Discoidin domain receptors: potential actors and targets in Cancer. Front Pharmacol. 2016;7:55

21. Ruiz PA, Jarai G. Collagen I induces discoidin domain receptor (DDR) 1 expression through DDR2 and a JAK2-ERK1/2-mediated mechanism in primary human lung fibroblasts. J Biol Chem. 2011;286(15):12912-23.
22. Ouyang F, et al. HMGB1 induces apoptosis and EMT in association with increased autophagy following $\mathrm{H} / \mathrm{R}$ injury in cardiomyocytes. Int J Mol Med. 2016;37(3):679-89.

23. Zhang $\mathrm{H}$, et al. PDGF-D/PDGFRbeta promotes tonque squamous carcinoma cell (TSCC) progression via activating p38/AKT/ERK/EMT signal pathway. Biochem Biophys Res Commun. 2016;478(2):845-51.

24. McCubrey JA, et al. Involvement of p53 and Raf/MEK/ERK pathways in hematopoietic drug resistance. Leukemia. 2008;22(11):2080-90.

\section{Ready to submit your research? Choose BMC and benefit from:}

- fast, convenient online submission

- thorough peer review by experienced researchers in your field

- rapid publication on acceptance

- support for research data, including large and complex data types

- gold Open Access which fosters wider collaboration and increased citations

- maximum visibility for your research: over $100 \mathrm{M}$ website views per year

At BMC, research is always in progress.

Learn more biomedcentral.com/submissions 